\title{
Mammalian sterile-like 1 kinase inhibits TGF $\beta$ and EGF-dependent regulation of invasiveness, migration and proliferation of HEC-1-A endometrial cancer cells
}

\author{
SANAZ ATTARHA ${ }^{1,2}$, SONIA ANDERSSON ${ }^{2}$, MIRIAM MINTS $^{2}$ and SERHIY SOUCHELNYTSKYI ${ }^{1}$ \\ Departments of ${ }^{1}$ Oncology-Pathology and ${ }^{2}$ Women's and Children's Health, \\ Karolinska Institutet, Stockholm, SE-17176, Sweden
}

Received March 20, 2014; Accepted April 16, 2014

DOI: $10.3892 /$ ijo.2014.2447

\begin{abstract}
Transforming growth factor- $\beta$ (TGF $\beta$ ) and epidermal growth factor (EGF) are two potent regulators of tumorigenesis. Signaling cross-talk of TGF $\beta$ and EGF employs a number of regulators which define the impact on cell physiology. MST1 has recently been reported as a regulator of tumorigenesis and differentiation. To investigate the role of mammalian sterile-like 1 (MST1) in TGF $\beta$ and EGF signaling, we established transiently MST1-transfected HEC-1-A endometrial cancer cells, and subjected the cells to treatment with TGF $\beta 1$, EGF and their combination. We report MST1 as a negative regulator of combined TGF $\beta$ and EGF signaling. We observed that enhanced expression of MST1 inhibited the combined action of TGF $\beta 1$ and EGF on cell invasiveness, migration and proliferation. Monitoring of the intracellular regulatory proteins showed that MST1 contribution to the TGF $\beta$-EGF cross-talk may involve focal adhesion kinase and E-cadherin, but not activation of Smad2. Our data unveiled the role of MST1 as a negative feedback for TGF $\beta 1$ - and EGF-regulated cell invasiveness, migration and proliferation.
\end{abstract}

\section{Introduction}

Tumorigenesis is the result of malfunction of many genes, proteins and metabolites. Among these molecules, TGF $\beta$ and EGF have prominent places as strong regulators of tumori-

Correspondence to: Dr Serhiy Souchelnytskyi, Department of Oncology-Pathology, Z5:01, Karolinska University Hospital, Solna, SE-17 176, Stockholm, Sweden

E-mail: serhiy.souchelnytskyi@ki.se

Dr Miriam Mints, Department of Women's and Children's Health, H2:0, Karolinska University Hospital, Solna, SE-171 76, Stockholm, Sweden

E-mail: miriam.mints@ki.se

Key words: mammalian sterile-like 1 kinase, transforming growth factor- $\beta$, epidermal growth factor, cell migration, cell invasiveness genesis. TGF $\beta$ has a double role in tumorigenesis. In the early stage of cancer, TGF $\beta$ has a tumor suppressor role which results in growth inhibition, cell cycle arrest and apoptosis. In the advanced stage of cancer, TGF $\beta$ promotes tumorigenesis. The cancer cells may lose responsiveness to TGF $\beta$ and may acquire aberrant TGF $\beta$ signaling, followed by promotion of survival, proliferation, EMT and increased motility and invasiveness of the cells (1). Numerous signaling pathways converge with the TGF $\beta$ pathway to modulate its effects; including signaling induced by EGF (2). EGF has predominately pro-mitogenic role in tumorigenesis (3). EGF promotes also cell survival, angiogenesis and differentiation. Deregulation of EGF pathways by overexpression or constitutive activation can promote tumorigenesis, including angiogenesis and metastasis, and is associated with a poor prognosis in many human malignancies (4). TGF $\beta$ and EGF intracellular signaling involves sharing intracellular signaling mechanisms. The extensive cross-talk between TGF $\beta$ and EGF involves such proteins and genes such as Smads, Erk1/2, p38 and PI3K.

Mammalian sterile-like 1 (MST1), a serine/threonine kinase of the sterile 20-like superfamily, has been reported to be a stress-activated protein participating in a wide range of apoptotic responses $(5,6)$. MST1 plays also an important role in mammalian development, cell cycle progression and tumorigenesis (7). Loss or reduction of MST1 expression has been observed in head and neck squamous cell carcinoma (8), soft tissue sarcoma (9), glioblastoma (10) and colorectal cancers (6), along with poorer cancer prognosis (9). Other in vivo studies have also indicated that the conditional ablation of MST1 resulted in liver enlargement (11). Despite the recent advance of our knowledge on the role of MST1 in tumorigenesis, the involvement of MST1 in other signaling pathway and regulation of invasiveness of endometrial cancer cells is unexplored. We report here that MST1 modulates cross-talk between TGF $\beta$ and EGF in regulation of cell proliferation, migration and invasiveness.

\section{Materials and methods}

Cell culture. HEC-1-A cells were obtained from American Type Culture Collection (Manassas, VA) and were cultured in McCoy's 5A Modified Medium supplemented with $10 \%$ fetal 
bovine serum, 1\% penicillin/streptomycin (Sigma-Aldrich, St. Louis, MO). Human recombinant TGF $\beta 1$ and EGF were obtained from PeproTech (Rocky Hill, NJ).

Cell transfection. A day before transfection HEC-1-A cells were cultured to $50 \%$ confluence for transfection. HEC-1-A cells were transfected with pcDNA3 control vector, wild-type and Ser82Ala mutant MST1-3XFLAG in pRC/CMV10 in 12-well plates using GeneJuice ${ }^{\circledR}$ transfection reagent, as recommended by the supplier (Novagen, Darmstadt, Germany). The expression constructs were kindly provided by Dr Zengqiang Yuan. Medium was changed $6 \mathrm{~h}$ after transfection and cells were incubated in complete medium for $48 \mathrm{~h}$ prior to treatments.

Cell proliferation assay. Cell proliferation in response to TGF $\beta 1$ and EGF treatment was measured by using CellTiter $96^{\circledR}$ AQueous One Solution Cell assay (Promega) according to the manufacturer's recommendations. Cells were grown in McCoy's 5A Modified Medium supplemented with $10 \%$ FBS and $1 \%$ penicillin/streptomycin with or without TGF $\beta 1(5 \mathrm{ng} / \mathrm{ml})$ and EGF $(10 \mathrm{ng} / \mathrm{ml})$ for $24 \mathrm{~h}$.

Cell apoptosis assay. Cell apoptosis was determined by using Cell Death Detection ELISAPlus (Roche, Germany). Briefly, cell lysates were placed in a streptavidin-coated microplate. A mixture of anti-histone-biotin and anti-DNA-peroxidase (anti-DNA-POD) was added and incubated for $2 \mathrm{~h}$ at $25^{\circ} \mathrm{C}$. After removal of unbound antibodies by washing steps, POD was determined photometrically at $405 \mathrm{~nm}$ with ABTS as substrate.

Wound healing assay. Cells were grown in culture media containing $10 \%$ of fetal bovine serum for $48 \mathrm{~h}$, until the cells reached the confluence. Monolayers of confluent cultures were scratched with a $20 \mu$ l-pipette tip, and images of the areas of scratches were taken under a microscope. TGF $\beta 1(5 \mathrm{ng} / \mathrm{ml})$ and EGF $(10 \mathrm{ng} / \mathrm{ml})$ were added, and the cells were incubated for $24 \mathrm{~h}$. After $24 \mathrm{~h}$, images of the areas of scratches were taken. Quantification was done by measuring the open wound area, which is the fraction of open image area at a later timepoint compared to the initial time-point, given as a percentage using TScrach software (12).

Migration assay. Cells were seeded on the membranes of wells of the 96-well plate $\mathrm{ChemoTx}^{\circledR}$ chemotaxis system (cat. no. \#116-8; Neuro Probe Inc.) in a culture medium containing growth factors as indicated for treatments. After $24 \mathrm{~h}$, the membrane was washed twice in PBS and fixed with $70 \%$ ethanol. The non-migrated cells were removed by cotton swab from the upper side of the membrane. Membrane was stained with $0.5 \%$ crystal violet, and subsequently visualized and quantified by using ImageJ software (13).

Invasion assay. Membranes of the 96-well plate ChemoTx chemotaxis system (cat. no. \#116-8) were covered with $3 \%$ gelatin, and cells were seeded on the membranes in a culture medium containing $5 \mathrm{ng} / \mathrm{ml}$ TGF $\beta 1$ and $10 \mathrm{ng} / \mathrm{ml}$ EGF. After $24 \mathrm{~h}$, the membrane was washed twice in PBS and fixed with $70 \%$ ethanol. The non-invaded cells were removed by cotton swab from the upper side of the membrane. Membrane was stained with $0.5 \%$ crystal violet, and subsequently visualized and quantified by using ImageJ software (13).

Immunoblot analysis. Cell lysates were resolved in 10\% SDS polyacrylamide mini-gels (Mini-protein Tetra Cell, Bio-Rad) and transferred onto nitrocellulose membranes (Whatman, Protran, Dassel, Germany). Membranes were blocked with $5 \%(\mathrm{~W} / \mathrm{V}) \mathrm{BSA}$ in TBS-T for $1 \mathrm{~h}$, and incubated with primary antibodies against target proteins at dilution recommended by the manufacturer, and followed by incubation with HRP-conjugated secondary antibody (GE Healthcare, Uppsala, Sweden). The proteins were visualized using Western Blotting Luminol Reagents (Santa Cruz Biotechnology Inc.). The antibodies used were: MST1 (AP7922a; Abgent), Flag M5, Smad2/3 (sc-6032), pSmad2 (sc-135644), anti-ERK1/2 (sc-135900), pERK1/2 (sc-7383), Rb (sc-50), pRb (sc-12901), vimentin (sc-7557), E-cadherin (sc-7870), FAK (sc-558), actin (sc-8432) all from Santa Cruz Biotechnology Inc., and anti-FAK (phospho Y397) (ab4803; Abcam).

Systemic analysis. Systemic analysis was performed with use of Cytoscape. GO terms of MST1, TGF $\beta 1$ and EGF were uploaded to generate a network, with inclusion of neighbors of the uploaded proteins, and databases were explored by Cytoscape plug-in MiMi.

Statistical analysis. Statistical significance of observed differences was evaluated using Mann-Whitney test among unpaired groups and among multiple groups by the Kruskal-Wallis test followed by Dunn's Multiple Comparison Test. Analysis were conducted using Graph Prism 6 software (GraphPad Software, San Diego, CA) and a $\mathrm{P}<0.05$ was considered significant.

\section{Results}

Expression of MST1 in HEC-1-A human endometrial cells. We identified earlier MST1 as a protein deregulated in endometrial cancer (14). Network analysis suggested that MST1 may be involved in a cross-talk between TGF $\beta$ and EGF (Fig. 1A). The involvement of MST1 may have an impact on cell proliferation via regulators of Ras and on cell death via regulation of caspase-3 and p53 (Fig. 1A). It has to be noted that molecular mechanisms of MST1 action are under extensive exploration, and therefore we may expect further interactions between MST1, TGF $\beta$ and EGF. However, available data are sufficient to suggest that MST1 may be involved in TGF $\beta$ and EGF cross-talk. Therefore, to explore the role of MST1 in responsiveness of cells to TGF $\beta 1$ and EGF, we used overexpression of the wild-type and Ser82Ala mutant of MST1 in human endometrial carcinoma HEC-1-A cells. The mutant MST1 was reported to have strongly decreased phosphorylation and disrupted dimerization (15). Both MST1 constructs were expressed to similar levels, and their expression was not affected by treatment of the cells with TGF $\beta 1$ and EGF alone or in combination (Fig. 1B). This indicates that TGF $\beta 1$ and EGF did not affect stability of the MST1 expressed in HEC-1-A cells. MST1-transfected cells showed no signs of enhanced cell death, indicating that MST1 itself under the used conditions did not induce cell death. 

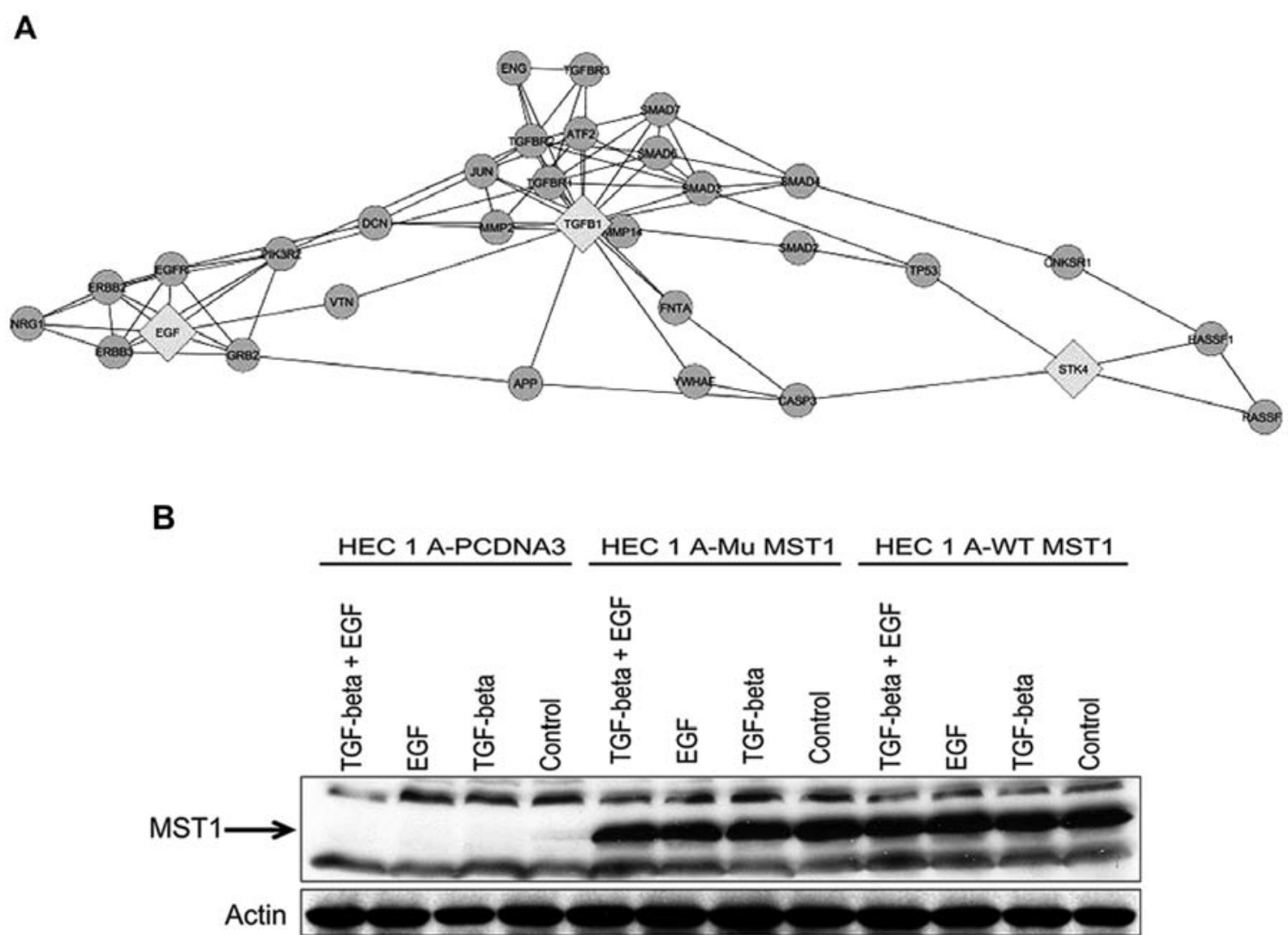

Figure 1. MST1 is a potential component of TGF $\beta$ and EGF cross-talk. (A) Network for MST1 proposed in the context of our previous proteomics study (14) is shown. Links between components define functional interactions. (B) Expression of wild-type and Ser82Ala mutant MST1 upon expression in HEC-1-A cells and treatment with TGF $\beta 1$, EGF and their combination. Migration positions of MST1 constructs are indicated. As a loading control, expression of actin was monitored.

Therefore, HEC-1-A cells transfected with the wild-type and mutant MST1 were used for further study.

Impact of MST1 on cell proliferation. First, we explored whether MST1 may affect cellular physiology and response to TGF $\beta 1$ and EGF in regulation of cell proliferation, apoptosis, migration and invasiveness. To study cell proliferation we performed MTT assay. TGF $\beta 1$ and/or EGF reduced the proliferation rate of cells (Fig. 2). The inhibitory effect of EGF was unexpected, but it was reproducible. The MST1 effect was rather marginal when the cells were treated with TGF $\beta 1$ or EGF only. The wild-type MST1 inhibited MTT activity in non-treated cells, but this effect was not observed when cells were treated with TGF $\beta 1$ or EGF. The inhibitory effect of combined treatment with TGF $\beta 1$ and EGF was prevented in cells expressing WT or mutant MST1 (Fig. 2). An interesting observation is that the MST1 mutant had similar impact as compared to the wild-type construct. These results indicate that the mutation of Ser82 in MST1 is not essential for the MST1 negative impact on TGF $\beta 1$ and EGF cross-talk (Fig. 2).

MST1 does not affect apoptosis of HEC-1-A cells. One of the cellular mechanisms in cancer is cell death. Therefore, we studied weather MST1 kinase affects cell apoptosis.
Expression of MST1 promoted apoptosis activity of the cells upon treatment with EGF and TGF 1 , and showed the stimulatory tendency for non-treated cells (Fig. 3). However, these effects were not strong, and did not affect cellular growth. Therefore, we concluded that the effect of MST1 on the cells death was not pronounced (Fig. 3).

MST1 affects cell migration regulated by TGF $\beta 1$ and EGF. To explore the effects of MST1 on cell migration, we performed wound healing and membrane migration assays. These assays explore cells in different conditions, e.g., in a confluent monolayer and in a sparse culture. However, in both assays the cells are prompted to migrate and this migration is then measured.

Wound healing assay showed that expression of MST1 constructs inhibited cell migration in non-treated and TGF $\beta 1$ or EGF treated cells (Fig. 4). When cells were treated with both TGF $\beta 1$ and EGF, a significant inhibition was observed (Fig. 4). This inhibition was slightly counteracted upon expression of the wild-type MST1. The most pronounced effect of TGF $\beta 1$ and EGF treatment on cell migration was observed in a membrane migration assay. We observed that combined treatment with TGF $\beta 1$ and EGF strongly induced cell migration, while single treatments had only marginal effect (Fig. 5). Expression of both MST1 constructs resulted in much lower migration rate of the cells. 


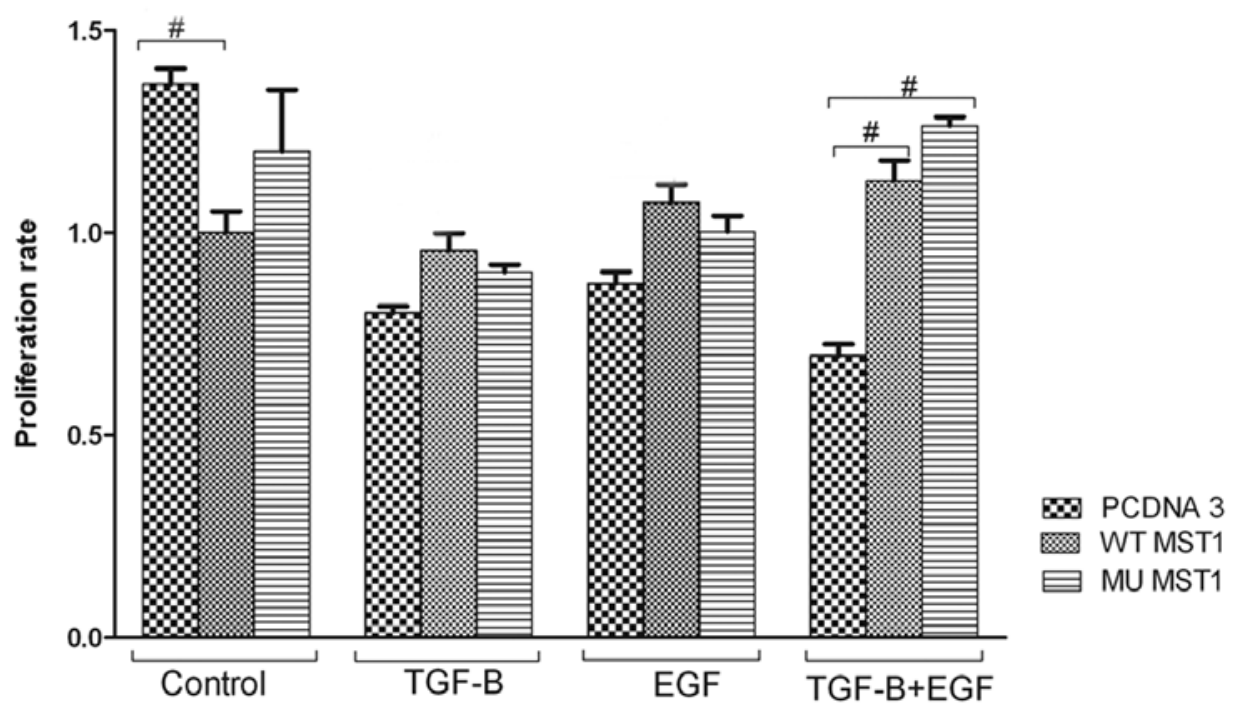

Figure 2. The MST1 impact on TGF $\beta 1$ and EGF-dependent regulation of cell proliferation. Treatments of cells transfected with the empty vector (HEC-1-A/PCDNA3), wild-type MST1 (HEC-1-A/WT MST1), and mutant MST1 (HEC-1-A/MU MST1) with TGF 31 , EGF and their combination are indicated. ${ }^{\#} \mathrm{p}<0.05$, for indicated conditions. Results of a representative MTT assay out of 3 performed are shown.

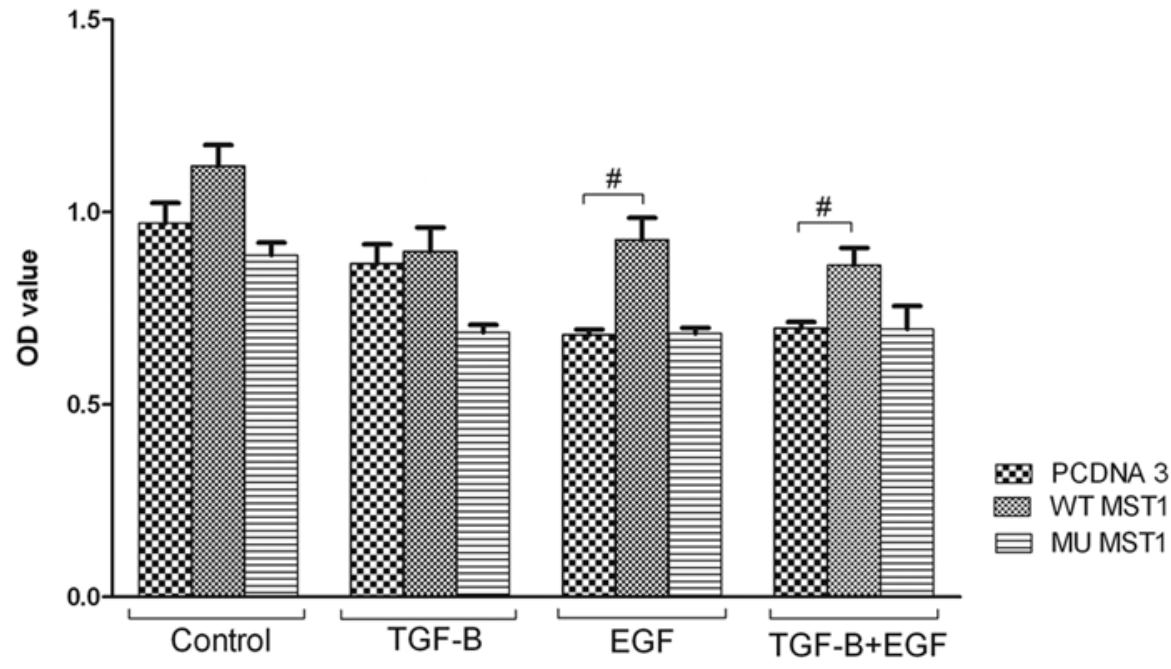

Figure 3. MST1 has a marginal effect on HEC-1-A cell death. Treatments of cells transfected with the empty vector (HEC-1-A/PCDNA3), wild-type MST1 (HEC-1-A/WT MST1), and mutant MST1 (HEC-1-A/MU MST1) with TGF $\beta 1$, EGF and their combination are shown. Results of a representative assay are shown.

Mutant MST1 significantly increases cell invasiveness. Invasiveness of cells is one of the features with high importance for tumorigenesis. We explored invasiveness of the cells through a layer of denaturated collagen, i.e., gelatin. We observed that transfection of MST1 constructs led to enhanced invasiveness of cells, although the level of induction was different under different treatment conditions (Fig. 6). The strongest stimulatory effect was observed upon expression of the mutant MST1 in non-treated cells. This stimulatory effect was abolished under treatment of cells with TGF $\beta 1$ and/or EGF. We observed that combined treatment of cells with TGF $\beta$ and EGF was decreased upon expression of the MST1 constructs. This observation indicates that the abrogation of dimerization and subsequent inhibition of the kinase activity of MST1 was not essential when cells were treated, but was of importance for response of non-treated cells (Fig. 6). Thus, MST1 constructs counteracted stimulatory effect on cell invasiveness of the combined treatment with TGF $\beta 1$ and EGF.

MST1 modulation of invasiveness correlates with phosphorylation of FAK, but not with phosphorylation of Smad2, Erk1/2, $p R b$ or expression of vimentin and E-cadherin. TGF $\beta$ and EGF employ many different signal transducers, with convergence on many common targets. To explore at which level of the signal transduction MST1 may interfere with TGF $\beta$ and 

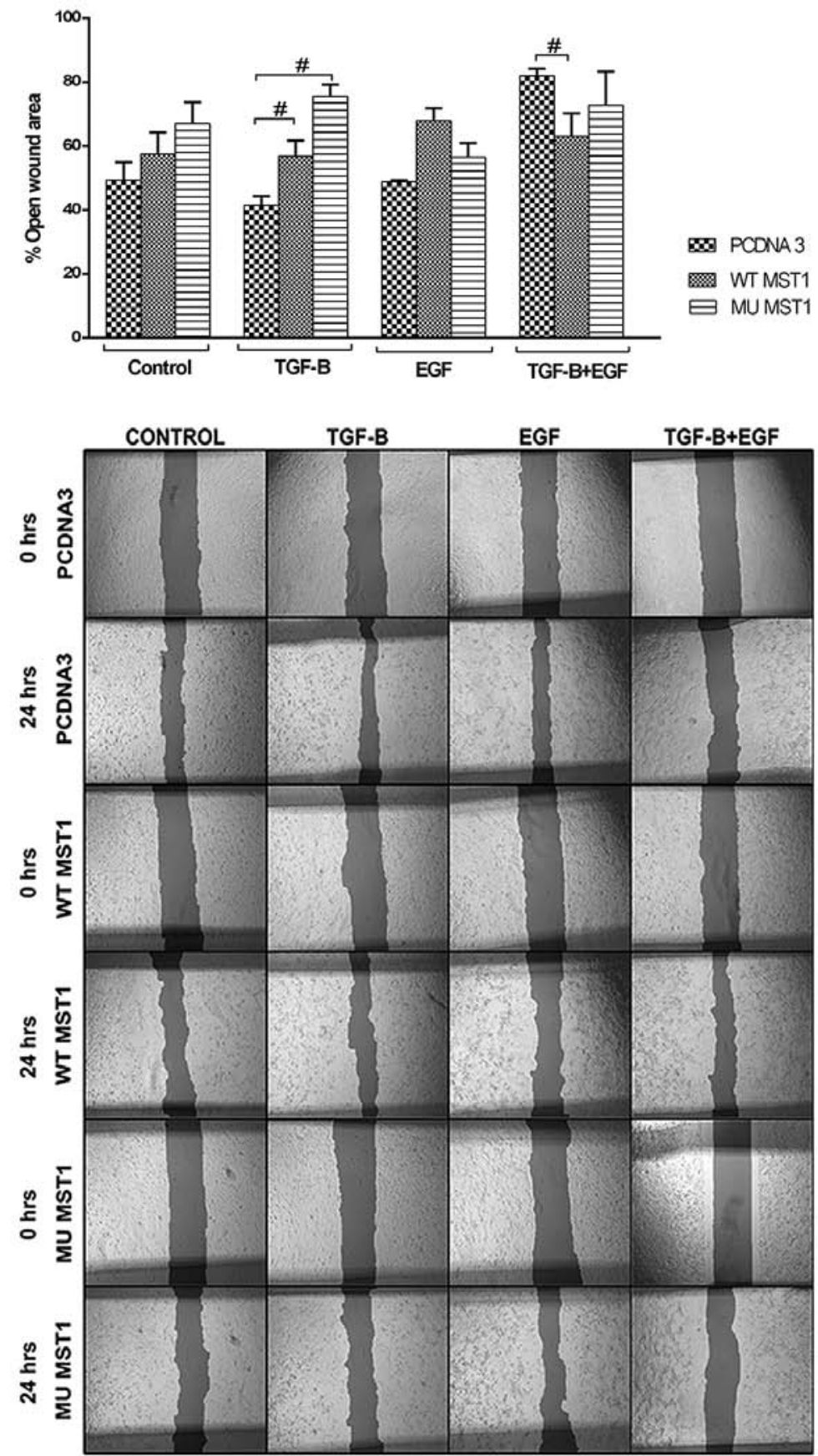

Figure 4. The MST1 effect on the cell migration in wound healing assay. The upper graph shows quantification of the results obtained in a wound healing assay. Examples of the assay images are shown in the lower part. Treatments of cells transfected with the empty vector (HEC-1-A/PCDNA3), wild-type MST1 (HEC-1-A/WT MST1), and mutant MST1 (HEC-1-A/MU MST1) with TGF 31 , EGF and their combination are shown. Results of a representative assay out of 3 performed are shown.

EGF signaling, we measured expression and/or activation of $\mathrm{Smad} 2, \mathrm{pRb}, \mathrm{FAK}$, Erk1/2 and expression of vimentin and E-cadherin.

Phosphorylation of Smad2 at its C-terminal serine residues reflects activation of signaling downstream of TGF $\beta$ receptors (16). We observed that only expression of the wild-type MST1 had a significant inhibitory effect on expression of Smad2 and Smad3, and phosphorylation of Smad2 (Fig. 7). In all other conditions, effects on Smads were weak or not significant. This indicates that MST1 does not affect TGF $\beta$ signaling events via Smad2 protein.
We observed no significant effect on $\mathrm{pRb}$ expression and its phosphorylation on Serine780 residue in cells treated as shown in Fig. 7. This observation is not in line with the results of the MTT proliferation assay (Fig. 2), and suggests that $\mathrm{pRb}$ expression and Ser780 phosphorylation did not correlate with the MST1 impact. Phosphorylation of Erk1/2 kinase correlates often with the proliferation rate of cells. As in the case with $\mathrm{pRb}$, we did not observe a correlation of Erk1/2 phosphorylation (Fig. 7) with the results of the proliferation assay (Fig. 2). However, the expression of the MST1 constructs did modulate Erk1/2 phosphorylation upon 


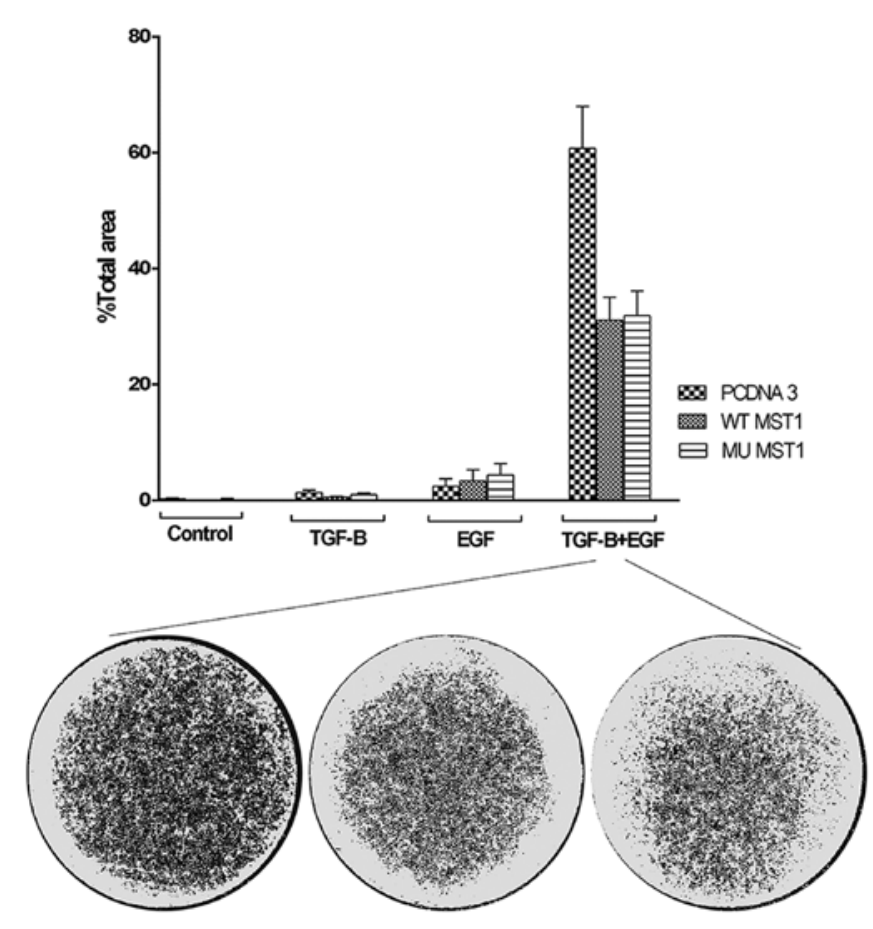

Figure 5. MST1 inhibits migration of cells induced by combined TGF $\beta 1$ and EGF. Columns show the total area of the membranes occupied by the cells which migrated through the membrane for HEC-1-A/PCDNA3, HEC-1-A/WT MST1 and HEC-1-A/MU MST1 cells treated with TGF 1 , EGF and their combination, as indicated. Images show examples of membranes with stained cells. A representative experiment out of 3 is shown.

treatments with TGF $\beta 1$ and/or EGF. This suggests that Erk1/2 phosphorylation is modulated by MST1, but this impact is not reflected in the proliferation rates of the cells.

Measuring phosphorylation and expression of focal adhesion kinase (FAK) allows monitoring cytoskeleton rearrangements involved in cell migration. We observed that FAK phosphorylation correlated with enhanced invasiveness of HEC-1-A cells transfected or not with MST1, and treated with different combinations of TGF $\beta 1$ and EGF (Fig. 7). For the results of migration assay, correlation was observed for all conditions, except the non-transfected cells which showed high phosphorylation of FAK, while no migration through the membrane was observed (Fig. 5).

E-cadherin and vimentin are markers of epithelial-mesenchymal phenotype of cells. They are also used as markers to evaluate invasiveness-related epithelial-mesenchymal transition (EMT). HEC-1-A cells show detectable expression levels of vimentin and E-cadherin (Fig. 7). Expression of either of MST1 constructs did not modulate vimentin expression while expression of the wild-type MST1 reduced E-cadherin levels upon single TGF $\beta 1$ and double TGF $\beta 1$ and EGF treatments. TGF $\beta$-dependent inhibition of E-cadherin expression is known as a part of TGF $\beta$-induced EMT. The results of immunoblot analysis for E-cadherin indicate that MST1 has modulatory impact on TGF $\beta$ and/or EGF regulated expression of E-cadherin, but this impact has to be combined with other regulatory processes, which then would result in an impact on cell proliferation, migration and invasiveness. Among the markers of intracellular signaling pathways, studied by us,

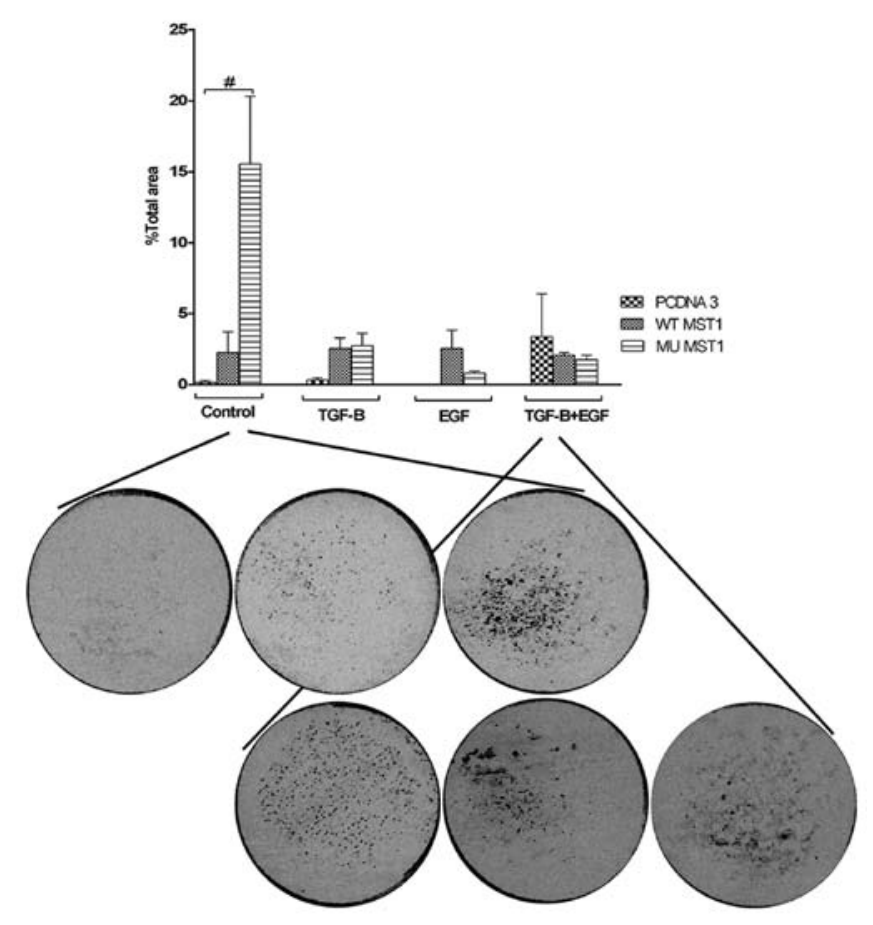

Figure 6. MST1 stimulated invasiveness of non-treated cells, but inhibited cells when treated with TGF $\beta 1$ and EGF. Columns show the total area of the membranes occupied by the cells which migrated through the membrane for HEC-1-A/PCDNA3, HEC-1-A/WT MST1 and HEC-1-A/MU MST1 cells treated with TGF $\beta 1$, EGF and their combination, as indicated. Images show examples of membranes with stained cells. The upper images show nontreated cells, and the lower images show cells under double treatment with TGF $\beta 1$ and EGF.

phosphorylation of FAK showed correlation with the pattern of cell invasiveness.

\section{Discussion}

Cellular functions are controlled by combinations of different regulators. Here we described the impact of MST1 on functional interaction between TGF $\beta$ and EGF in regulation of cell invasiveness, migration and proliferation. Recent studies showed that MST1 regulates cell death, differentiation and proliferation (17). Aberrations in MST1 expression have been observed in tumorigenesis, with indication that MST1 may have a tumor suppressive role $(6,18)$. Mechanisms of MST1 intracellular signaling mechanisms are currently under investigation in many laboratories, and one of the key conclusions is that MST1 may play role of a coordinator between different pathways.

EGF and TGF $\beta$ are two well-studied regulators of tumorigenesis. EGF is predominantly tumor-promoting factor, due to its strong pro-mitogenic activity (3). TGF $\beta$ on the contrary is a strong inhibitor of proliferation of epithelial cells. However, TGF $\beta$ has a double role in tumorigenesis $(19,20)$. At the early stages it inhibits tumor growth, while at the later stages it promotes metastases. Extensive cross-talk between EGF and TGF $\beta$ has been described. Intracellular regulators, which first were considered as specific for TGF $\beta$ or EGF pathways, later were shown to be shared between these pathways $(19,20)$. 

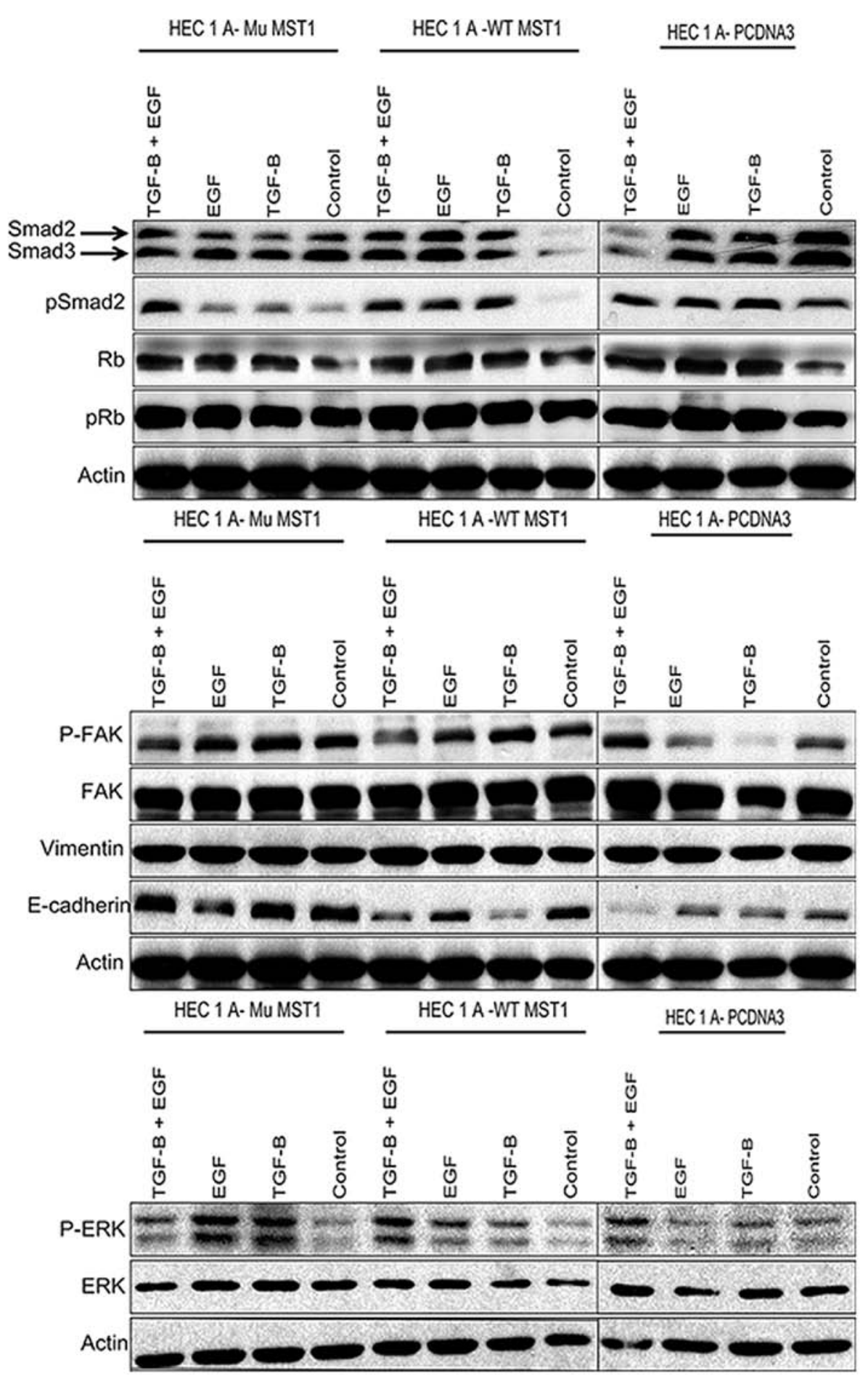

Figure 7. Impact of MST1 on expression and phosphorylation of intracellular proteins. Expression and phosphorylation of Smad2, pRb, FAK and Erk1/2, and expression of vimentin and E-cadherin in cells transfected with the wild-type (WT MST1) or mutant (Mu MST1) MST1, and treated as indicated are shown. Immunoblot analysis for actin was used to monitor equal loading. Migration positions of tested proteins are annotated.

Identification of MST1 as a protein with potential involvement in cross-talk of TGF $\beta$ and EGF (Fig. 1A) prompted us to explore whether MST1 indeed affects EGF and TGF $\beta$ dependent regulation of cell invasiveness, migration and proliferation. MST1 has been reported to induce cell death $(10,21,22)$. We observed only marginal cell death induction upon transfection of the wild-type MST1 in HEC-1-A cells (Fig. 3). Our results showed that MST1 may act as a negative regulator of combined action of TGF $\beta$ and EGF on cell invasiveness and migration, while its effect is not pronounced when cells are challenged with each of the growth factors separately (Figs. 5 and 6). This observation underlines the importance of exploring combinatorial treatments. The challenge of such exploration is in the high number of intracellular regulators which have to be tested. We monitored some of the proteins which may reflect activation of TGF $\beta$ 
and EGF signaling, i.e., Smad2 and Erk1/2, and proteins reflecting migratory and invasive mechanisms, i.e., FAK, vimentin and E-cadherin (Fig. 7). The reported exploration of the cellular responses to the combination of TGF $\beta$, EGF and MST1, together with the evaluation of protein markers of signaling pathways provide a rationale for further more elaborate mechanistic studies. Our data include also MST1 in the network of TGF $\beta$ and EGF signaling, which may improve prediction of responses to EGF and TGF $\beta$ targeting drugs already used or in clinical trials for treatment of cancer.

\section{Acknowledgements}

We are grateful to Dr Zengqiang Yuan for the gift of the MST1 constructs. We are also grateful to the Oves Minnesfond for support and encouragement. This study is supported in part by grants from the Radiumhemmet research funds (\#121202), the Swedish Cancer Society, the Swedish Research Council, the Swedish Institute, INTAS, Erasmus KI-UWM and STINT to S.S., and grants from the regional agreement on medical training and clinical research (ALF) between Stockholm County Council and the Karolinska Institute and Swedish Labour Market Insurance (AFA) to M.M.

\section{References}

1. Jakowlew SB: Transforming growth factor-beta in cancer and metastasis. Cancer Metastasis Rev 25: 435-457, 2006.

2. Dunfield LD and Nachtigal MW: Inhibition of the antiproliferative effect of TGFbeta by EGF in primary human ovarian cancer cells. Oncogene 22: 4745-4751, 2003.

3. Scaltriti $\mathrm{M}$ and Baselga J: The epidermal growth factor receptor pathway: a model for targeted therapy. Clin Cancer Res 12 5268-5272, 2006.

4. Lurje G and Lenz H-J: EGFR signaling and drug discovery. Oncology 77: 400-410, 2009.

5. Creasy CL and Chernoff J: Cloning and characterization of a member of the MST subfamily of Ste20-like kinases. Gene 167: 303-306, 1995.

6. Minoo P, Zlobec I, Baker K, Tornillo L, Terracciano L, Jass JR and Lugli A: Prognostic significance of mammalian sterile20-like kinase 1 in colorectal cancer. Mod Pathol 20 331-338, 2007.
7. Cinar B, Collak FK, Lopez D, et al: MST1 is a multifunctional caspase-independent inhibitor of androgenic signaling. Cancer Res 71: 4303-4313, 2011.

8. Steinmann K, Sandner A, Schagdarsurengin U and Dammann RH: Frequent promoter hypermethylation of tumor-related genes in head and neck squamous cell carcinoma. Oncol Rep 22: 1519-1526, 2009.

9. Seidel C, Schagdarsurengin U, Blümke K, et al: Frequent hypermethylation of MST1 and MST2 in soft tissue sarcoma. Mol Carcinog 46: 865-871, 2007.

10. Qiao M, Wang Y, Xu X, et al: Mst1 is an interacting protein that mediates PHLPPs' induced apoptosis. Mol Cell 38: 512-523, 2010.

11. Song H, Mak KK, Topol L, et al: Mammalian Mst1 and Mst2 kinases play essential roles in organ size control and tumor suppression. Proc Natl Acad Sci USA 107: 1431-1436, 2010.

12. Gebäck T, Schulz MMP, Koumoutsakos P and Detmar M: TScratch: a novel and simple software tool for automated analysis of monolayer wound healing assays. Biotechniques 46: 265-274, 2009.

13. Schneider CA, Rasband WS and Eliceiri KW: NIH Image to Image J: 25 years of image analysis. Nat Methods 9: 671-675, 2012.

14. Attarha S, Andersson S, Mints M and Souchelnytskyi S: Individualised proteome profiling of human endometrial tumours improves detection of new prognostic markers. Br J Cancer 109: 704-713, 2013.

15. Bi W, Xiao L, Jia Y, et al: c-Jun N-terminal kinase enhances MST1-mediated pro-apoptotic signaling through phosphorylation at serine 82. J Biol Chem 285: 6259-6264, 2010.

16. Souchelnytskyi S, Tamaki K, Engström U, Wernstedt C, ten Dijke P and Heldin CH: Phosphorylation of Ser465 and Ser467 in the C terminus of Smad2 mediates interaction with Smad4 and is required for transforming growth factor-beta signaling. J Biol Chem 272: 28107-28115, 1997.

17. Qin F, Tian J, Zhou D and Chen L: Mst1 and Mst2 kinases: regulations and diseases. Cell Biosci 3: 31, 2013.

18. Ng Y-K, Lau W-S, Lui VWY, et al: Full-length Mst1 exhibits growth promoting function in human hepatocellular carcinoma cells. FEBS Lett 587: 496-503, 2013.

19. Katsuno T, Ochi M, Tominaga K, et al: Mesenchymal stem cells administered in the early phase of tumorigenesis inhibit colorectal tumor development in rats. J Clin Biochem Nutr 53: 170-175, 2013.

20. Massagué J: TGF- $\beta$ signaling in development and disease. FEBS Lett 586: 1833, 2012.

21. Graves JD, Gotoh Y, Draves KE, et al: Caspase-mediated activation and induction of apoptosis by the mammalian Ste20-like kinase Mst1. EMBO J 17: 2224-2234, 1998.

22. Lin Y,Khokhlatchev A,Figeys D and Avruch J: Death-associated protein 4 binds MST1 and augments MST1-induced apoptosis. J Biol Chem 277: 47991-8001, 2002. 